Pensamiento Crítico No. 7, pag. 1 - 12

\title{
La Demanda de Educación Universitaria Estatal (DEUS) una Apreciación para el Periodo 1980-2006 \\ Ysac Ospino Edery
}

Resumen: La demanda por educación Universitaria Estatal representa toda una problemática, por su crecimiento Anárquico, donde miles postulan sin saber sin tienen los atributos requeridos para la carrera escogida, y otro tanto que egresa de la Universidad no consigue trabajo por encontrarse el mercado saturado de profesionales de la misma especialidad, por lo que se ven obligados a ser sub empleados o emigrar fuera del país en busca de dudosos horizontes.

Mercado que con mano invisible y espíritu liberal, va ordenando el que profesiones, como, cuando y donde deben fomentarse, mientras que el Ministerio de educación, la Asamblea nacional de rectores así como las principales autoridades Universitarias, no realizan mayor acción de carácter planificador orientado a solucionar los requerimientos reales que la sociedad para su desarrollo exige.

Demanda que ha venido siendo determinada en los últimos veinte años, entre otros factores por la concentración de la población en las grandes ciudades producto de las migraciones y por el deterioro del ingreso real de las familias, como se aprecia en los coeficientes de correlación elevados de Pearson resultantes, de (0.80) para la correlación postulantes a las Universidades Estatales Vs remuneración mínima vital y de (0.91) para la correlación postulantes a las Universidades Estatales Vs concentración de la población en las zonas urbanas. 


\section{Ysac Ospino Edery}

Palabras claves: Demanda por Educación Universitaria Estatal: es aquella constituida por todos los postulantes matriculados como tales que pretenden acceder a la Universidad Estatal.

Concurso de admisión: Sistema que establece la Universidad para la selección de sus postulantes a efectos de cubrir el número de plazas de vacantes que dispone.

Concentración de la población en las zonas urbanas: Núcleos de poblaciones compactas que habitan en las ciudades dedicados a ocupaciones no agrícolas

\section{Reflexiones para determinar problemática}

Hoy vemos por las calles de las diferentes ciudades, a miles de desempleados y subempleados, muchos de los cuales son profesionales en abogacía, administración, pedagogía, economía, educación etc., a los que se sumaran muchos otros que egresarán, de las Universidades estatales, privadas e institutos de educación superior, haciendo largas colas frente a empresas u otras organizaciones, cuando aparece algún aviso ofreciendo una vacante o frente a los consulados de diversos países pretendiendo que se les otorgue una visa para poder emigrar.

"En una década emigraron a otros países cerca de 30 mil profesionales de la salud peruanos", afirmó la asesora del Ministerio de salud (Minsa) Paulina Giusti quien preciso que aproximadamente mil 356 enfermeras y 12 mil 298 médicos han viajado a diversos países entre los años 1994 y 2004

Esta situación esta vinculada a los diversos aspectos, entre ellos la disociación entre el mundo del trabajo y el mundo de la formación en salud, caracterizada por el desajuste entre la oferta educativa y la demanda de los servicios, pues existe una notoria sobreoferta de educación en salud infomo" ".

Profesionales que al no encontrar una fuente de ingresos, a fin a sus estudios realizados o algún otro que le procure un sustento, se ven obligados a emigrar al extranjero, en busca del ansiado trabajo, desarraigándose, de sus familias, costumbres, cariños etc., donde posiblemente los catalogaron como lo ultimo del escalafón social (ilegales, indocumentados) de aquellos países que consideran como destino.

Emigración que implica la consecuente pérdida de la inversión en su educación para su familia que posiblemente la realizo postergando otras necesidades, y para el país la merma de 
La demanda de educación universitaria estatal (DEUS) una apreciación para el periodo 1980-2006

un capital humano apto, a lo que se agrega un sentido de frustración que no lograran superar en muchos años y otros nunca.

Situación contraproducente si apreciamos a los miles de postulantes que demandan por una vacante en las diferentes universidades para seguir la profesión que les acomode a sus requerimientos y creencias, a los cuales se les pone como condición superar en la mayoría de los casos exámenes muy exigentes de diversos conocimientos para ser admitidos.

Conocimientos a los que podrán acceder, luego de una preparación costosa en centros pre universitarios, que llega a mas de un año de duración en la mayoría de los casos, adicionales, a sus años de estudios, dado que la formación escolar resulta ser pobre e insuficiente, involucrando muchas horas de sacrificio y postergando el costo de oportunidad de aquellas actividades como, viajes, trabajos que se han tenido que dejar de lado.

Postulantes que demandarán una vacante en la mayoría de los casos, en las universidades del estado por lo económico que resulta obtener una formación profesional frente a lo costosas de las universidades privadas, tal como se aprecia en el Cuadro No. 1, tomando como referencia el costo (matrícula y pensión que paga un padre de familia), demanda, la misma que en los últimos años a tenido un crecimiento geométrico frente a una oferta de crecimiento aritmético de las vacantes ofrecidas.

Cuadro No. 1 : Matricula y pensión mensual año 2006. (en soles)

\begin{tabular}{|l|c|c|r|}
\hline \multicolumn{1}{|c|}{ DESCRIPCIÓN } & $\begin{array}{c}\text { MATRICULA } \\
\text { AL INICIO }\end{array}$ & $\begin{array}{c}\text { PENSION } \\
\text { MENSUAL }\end{array}$ & $\begin{array}{r}\text { TOTAL } \\
\text { ANUAL X 9 }\end{array}$ \\
\hline UNIVESIDAD NACIONAL MAYOR DE SAN MARCOS & & & 160.00 \\
\hline UNIVERSIDAD SAN MARTÍN DE PORRES (1) & 600.00 & 700.00 & 6300.00 \\
\hline UNIVERSIDAD PERUANA DE CIENCIAS (2) & 1000.00 & 900.00 & 8100.00 \\
\hline UNIVERSIDAD SAN IGNACION DE LOYOLA (3) & 300.00 & 1200.00 & 10800.00 \\
\hline
\end{tabular}

Fuente: Informacion obtenida de padres de familia 


\section{Ysac Ospino Edery}

La concentración de la población en las zonas urbanas - marginales; se constituye en una de las variables altamente influyentes en el comportamiento de la demanda del sistema educativo en todos sus niveles. La composición demográfica y la mayor escolaridad de la población exigen una reflexión profunda sobre la orientación del desarrollo futuro de las instituciones de educación superior, por parte de los organismos rectores correspondientes.

Las estimaciones del INEI señalan que el descenso de la mortalidad mantendría el ritmo que sigue el proceso de un mayor envejecimiento de la población, sino que esta disminución se aceleraría durante la primera mitad del presente siglo, como se aprecia en los cuadros No. 2,3y 4.

Cuadro No. 2 - Perú: Estructura por grupos de edad 1993 - 2005

\begin{tabular}{|c|c|c|}
\hline Grupo de Edad & $\mathbf{1 9 9 3}$ & $\mathbf{2 0 0 5}$ \\
\hline Población & $22^{\prime} 04$ & $27^{\prime} 9$ \\
\hline Edad & $\%$ & $\%$ \\
\hline $0-14$ & 37.0 & 32.2 \\
\hline $14-64$ & 58.4 & 62.6 \\
\hline 65 a mas & 4.6 & 5.2 \\
\hline
\end{tabular}

FUENTE: INEI - Censo y Población 1993 - Estimaciones de Población 1950 - 2005

Cuadro No. 3 : Esperanza de Vida al Nacer - 2005 (en años)

\begin{tabular}{|c|c|c|}
\hline Sexo & 1995 & 2005 \\
\hline Total & 66.9 & 70.5 \\
\hline Hombre & 64.6 & 68.5 \\
\hline Mujer & 69.4 & 73.3 \\
\hline
\end{tabular}

FUENTE: INEI Censo de Población 1993 - Estimaciones de Población 1950 - 2005

Cuadro No. 4 : Perú - Esperanza de Vida: 1950 - 2005

FUENTE: INEI - Estimaciones y proyecciones

\begin{tabular}{|c|c|}
\hline Periodos & Años \\
\hline $1950-55$ & 43.9 \\
\hline $1970-75$ & 55.9 \\
\hline $1990-95$ & 66.7 \\
\hline $1995-00$ & 68.3 \\
\hline $2000-05$ & 69.8 \\
\hline
\end{tabular}


La demanda de educación universitaria estatal (DEUS) una apreciación para el periodo 1980-2006

El cambio demográfico y la consiguiente modificación en la estructura por edades tendrán efectos relevantes en el sistema educativo en general y en la demanda de educación tanto de la población tradicionalmente demandante (de 20 a 24 años), como de la población de mayor edad.

Así uno de los retos fundamentales a enfrentar en los próximos años será el de desarrollar una infraestructura para atender a la creciente población escolar en los niveles de educación primaria, secundaria y superior. Este incremento esperado de gran magnitud en la matricula de los niveles mencionados, representan un reto a la imaginación y la capacidad innovadora educativa de los gobiernos de turno.

Lo urbano como otro elemento de análisis para el estudio de la DEUS, se encuentra en un proceso de acelerado crecimiento. Tenemos así que en la segunda mitad del siglo veinte, como producto de las migraciones, que tienen su origen en la búsqueda de mejores condiciones de vida (seguridad, educación, salud, trabajo, vivienda, etc) se constituyeron decenas de asentamientos humanos, que luego se transformaron en urbanizaciones y hoy en día, algunos son prósperos y pujantes distritos.

La educación es un requerimiento eminentemente urbano para el Perú, sus desequilibrios corresponden en gran medida a las distorsiones del crecimiento y distribución de nuestra población como se presenta en el cuadro siguiente No.5. Así tenemos que la concentración de la población en grandes ciudades delimita la demanda por educación superior, por lo que su desarrollo futuro será determinante en la evolución de la educación superior en las próximas décadas.

La correlación lineal resultante entre postulantes a las Universidades Estatales y la concentración de la población en la zonas urbanas con el coeficiente de correlación de Pearson nos da un relativo valor fuerte de (0.91) cercano a (1), indicador que contribuye con nuestra aseveración anterior.

Cuadro No. 5 : Perú - Concentración de la Población

\begin{tabular}{|l|c|c|c|c|c|c|c|}
\hline & \multicolumn{7}{|c|}{$\%$} \\
\hline Area & 1940 & 1961 & 1973 & 1985 & 1995 & 2000 & 2005 \\
\hline Urbana & 35.4 & 47.4 & 59.6 & 66.3 & 68.8 & 71.9 & 72.6 \\
\hline Rural & 64.6 & 52.6 & 40.5 & 33.7 & 31.2 & 28.1 & 27.4 \\
\hline
\end{tabular}

Fuente: INEI - Estimaciones y Proyecciones de Población 1950-2005 


\section{Ysac Ospino Edery}

Desde la perspectiva del sistema nacional de educación superior, se hace imperativo el estudiar de cerca las tendencias y proponer las medidas correctivas hacia un mejor reordenamiento de la población en el territorio nacional.

El deterioro continuo del ingreso real de las familias como resultante de las crisis económicas de los últimos 20 años para no ir mas lejos, aspecto que se hace manifiesto en el cuadro Nro 6, donde tenemos que la remuneración mínima vital real mensual alcanzo su menor nivel el año 1993, el de un monto de S/ 79.03 el cual resulta 10 veces inferior al de 1980, para luego iniciar un periodo de recuperación que al año 2006 todavía se ubica en un $38 \%$ del registrado para el año 1980.

Situación que ha determinado, entre otros factores a que una población empobrecida, busque en la educación superior universitaria estatal de algunos de sus miembros la posibilidad, alternativa, a la superación de su empobrecimiento al comprender que la constitución de un capital humano representa un medio entre otros, donde sabemos que las pequeñas diferencias educativas en el presente determinan también menores diferencias económicas, pero las mayores diferencias educativas en el mañana marcaran también mucho mayores distancias económicas y sociales, la que procurara una mejor salud, una vida prolongada, mayores comodidades materiales, y en consecuencia un mejor nivel de vida.

Podemos añadir a lo anterior, los centenares de institutos superiores (la mayoría negocios muy rentables) que se han constituido en las principales capitales como Huancayo, Huaraz, Huanuco, etc, que de alguna manera contribuyen a solucionar la demanda por educación superior de sus poblaciones que ven en la educación un camino para salir de su pobreza que no han podido superar por generaciones.

"A fines de las décadas de los 80' la pobreza estructural o permanente se suma a la pobreza (coyuntural) o reciente, derivada de las políticas de estabilización llevadas a cabo antes de 1990. Por ello la mitad de la población en el Perú se ubica bajo la línea de pobreza y un quinto de ella sé allá en extrema pobreza"

A lo que se agrega la dificultad de no contar con un plan nacional estable de desarrollo económico a mediano y largo plazo del sistema de educación superior estatal, sustentado en un real apoyo financiero a sus presupuestos.

Las Universidades publicas sobreviven año tras año con la amenaza constante de no contar con un presupuesto suficiente, mientras que las Universidades particulares se ven afectadas por la disminución de los ingresos de los sectores sociales que son demandantes de 
La demanda de educación universitaria estatal (DEUS) una apreciación para el periodo 1980-2006

ellas, habiéndose iniciado, campañas masivas de promoción, publicidad y competencia entre las mismas.

Cuadro No. 6

Remuneración Mínima Vital Real Mensual

Periodo Base 1994 en Nuevos Soles

\begin{tabular}{|l|l|l|}
\hline Años & Enero & Diciembre \\
\hline 1980 & 816.34 & 757.89 \\
1981 & 733.11 & 643.64 \\
1982 & 677.04 & 600.71 \\
\hline 1983 & 558.08 & 600.54 \\
1984 & 559.66 & 531.63 \\
1985 & 466.63 & 439.86 \\
\hline 1986 & 418.30 & 450.03 \\
1987 & 422.29 & 512.83 \\
1988 & 454.74 & 269.52 \\
\hline 1989 & 243.09 & 193.52 \\
1990 & 195.29 & 143.52 \\
1991 & 185.13 & 91.19 \\
\hline 1992 & 88.07 & 110.23 \\
1993 & 105.14 & 79.03 \\
1994 & 77.61 & 125.57 \\
\hline 1995 & 125.11 & 113.92 \\
1996 & 112.52 & 165.91 \\
1997 & 165.12 & 250.07 \\
\hline 1998 & 247.87 & 235.90 \\
1999 & 235.86 & 227.42 \\
2000 & 227.57 & 260.05 \\
\hline 2001 & 260.05 & 260.87 \\
2002 & 262.24 & 256.98 \\
2003 & 256.39 & 281.33 \\
\hline 2004 & 279.83 & 271.87 \\
2005 & 271.59 & 267.86 \\
2006 & 289.71 & 287.85 \\
\hline
\end{tabular}

Fuente : Ministerio de Trabajo y Promoción del Empleo Dirección Nacional de Empleo y Formación Profesional 


\section{Ysac Ospino Edery}

\section{La demanda para Educación Universitaria}

Apreciando el Gráfico 7 A y el Cuadro No. 7, tenemos que para 1985 se registraron 208,493 postulantes, 141,249 para las universidades públicas y 67,244 para las universidades privadas, para el año 2006 cifras preliminares registran 394,445 postulantes, lo que representa un incremento del $89.2 \%$, distribuido en un aumento del $107.6 \%$ y un $51.5 \%$ para las universidades públicas y privadas respectivamente, cifras que presenta una tendencia creciente de la demanda por educación superior universitaria estatal y privada.

\section{Gráfico 7 A.}

Universidades del Perú - Numero de postulantes 1960 -2004

TOTAL POSTULANTES

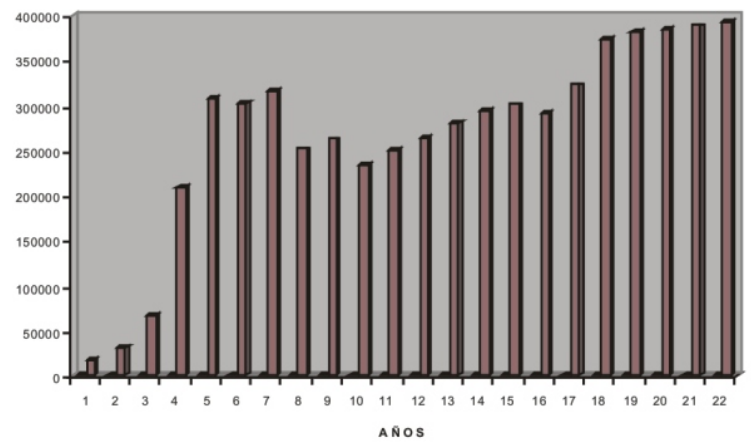

Cuadro No. 7

Universidades del Perú. Número de Postulantes 1960 - 2004

\begin{tabular}{|c|c|c|c|c|}
\hline & AÑOS & TOTAL & PÚBLICA & PRIVADA \\
\hline 1 & 1960 & 17,305 & $\mathrm{Nd}$ & $\mathrm{Nd}$ \\
\hline 2 & 1965 & 32,390 & $\mathrm{Nd}$ & $\mathrm{Nd}$ \\
\hline 3 & 1970 & 66,199 & $\mathrm{Nd}$ & $\mathrm{Nd}$ \\
\hline 4 & 1985 & 208,493 & 141,249 & 67,244 \\
\hline 5 & 1986 & 307,438 & 218,950 & 88,488 \\
\hline 6 & 1987 & 302,990 & 204,945 & 98,045 \\
\hline 7 & 1988 & 315,968 & 231,834 & 84,134 \\
\hline 8 & 1989 & 252,078 & 175,733 & 76,345 \\
\hline 9 & 1990 & 263,137 & 176,688 & 86,449 \\
\hline 10 & 1991 & 233,510 & 159,384 & 74,126 \\
\hline 11 & 1992 & 250,321 & 174,683 & 75,638 \\
\hline 12 & 1993 & 263,639 & 197,728 & 65,911 \\
\hline
\end{tabular}


La demanda de educación universitaria estatal (DEUS) una apreciación para el periodo 1980-2006

\begin{tabular}{|l|l|l|l|l|}
\hline 13 & 1994 & 280,426 & 217,755 & 62,671 \\
\hline 14 & 1995 & 293,597 & 226,892 & 66,705 \\
\hline 15 & 1996 & 301,551 & 237,187 & 64,364 \\
\hline 16 & 1997 & 291,256 & 229,107 & 62,149 \\
\hline 17 & 1998 & 323,553 & 245,504 & 78,049 \\
\hline 18 & 1999 & 373,393 & 301,191 & 72,202 \\
\hline 19 & 2000 & 381,447 & 312,914 & 68,533 \\
\hline 20 & 2004 & 384,208 & 285,697 & 98,511 \\
\hline 21 & 2005 & 389,318 & 290,042 & 99,276 \\
\hline 22 & 2006 & 394,495 & 293,307 & 19,188 \\
\hline
\end{tabular}

Tasa Promedio de crecimiento anual: $1.33 \%$

Fuente: Asamblea Nacional de Rectores Grade (Grupo de Análisis para el Desarrollo)

La correlación lineal obtenida con el coeficiente de Pearson, entre el quantum de postulantes a las Universidades Estatales y la remuneración mínima vital nos da un valor fuerte de (0.80), cercano a (1) para los últimos 20 años.

Apreciando el cuadro Nro 8 de indicadores varios se tiene que el indicador de (ingresos/postulantes x 100) al cual se denomina "tasa de ingreso", columna E, para el periodo comprendido entre al año 1970 y 2004, , ha fluctuado alrededor de un 30\%, con un $70 \%$ que no lograron ingresar lo que equivale a 255,800 postulantes para el año 2004 , situación que se ve agravada para el año 2006 y para años futuros dado el mayor número de postulantes esperado según la su tasa de crecimiento histórico del $1.33 \%$.

Cuadro No. 8

Indicadores varios

\begin{tabular}{|c|c|c|c|c|c|c|c|}
\hline Años & $\begin{array}{c}\text { Poblac. } \\
\text { Total A }\end{array}$ & $\begin{array}{c}\text { Posts. } \\
\text { B }\end{array}$ & $\begin{array}{c}\text { Posts. } \\
\text { Pob. C }\end{array}$ & $\begin{array}{c}\text { Ingresantes } \\
\text { D }\end{array}$ & $\begin{array}{c}\text { Ing./ } \\
\text { Postul. E }\end{array}$ & $\begin{array}{c}\text { Ing./ } \\
\text { Pob. F }\end{array}$ & $\begin{array}{c}\text { Ing-Postu } \\
\text { G }\end{array}$ \\
\hline 1970 & 13,192 & 66,199 & $5 \%$ & 23,914 & 36.1 & 0.18 & $(42,285)$ \\
\hline 1980 & 17,295 & 239,485 & $1.4 \%$ & 58,744 & 24.5 & 0.33 & $(180,741)$ \\
\hline 1990 & 21,550 & 263,137 & $1.2 \%$ & 73,243 & 27.8 & 0.33 & $(189,894)$ \\
\hline 2000 & 26,275 & 381,447 & $1.5 \%$ & 101,476 & 26.6 & 0.38 & $(279,971)$ \\
\hline 2004 & 26,800 & 384,208 & $1.5 \%$ & 128,408 & 33.4 & 0.48 & $(255,800)$ \\
\hline 2006 & nd & 394,495 & Nd & & & & \\
\hline
\end{tabular}

Fuente: INEI, Asamblea Nacional de Rectores 


\section{Ysac Ospino Edery}

El crecimiento mencionado (incremento de postulantes) se puede atribuir entre otras razones, al acelerado aumento demográfico de la población, a las mayores exigencias del mercado que requiere personal más diversificado con una mayor capacitación y competitividad.

La demanda por educación superior universitaria ha crecido de una manera desorganizada y anárquica, sin un "Plan de Desarrollo", de este sub sector, donde los postulantes solo quieren ingresar, sin mayormente saber si tienen las aptitudes necesarias para la profesión que han elegido o si al final cuando egresen puedan conseguir una oferta de trabajo.

Qué entendemos por Plan de Desarrollo, sino "el conjunto de previsiones y objetivos para cuya realización se reúnen formando una estructura coherente, medios definidos", buscando dar respuestas cuantitativas y cualitativas, a las preguntas que no solo son económicas sobre, qué profesiones fomentar, cómo fomentarlas, dónde fomentarlas y para quién fomentarlas, teniendo como fin al individuo como ser social, donde las universidades alcancen metas y objetivos de corto, mediano y largo plazo obedeciendo al interés nacional y no a los grupos políticos de turno.

\section{Problemática de la Demanda por Educación Universitaria Estatal}

El crecimiento desordenado y anárquico de la demanda, ha traído como lógica consecuencia que egresen miles de profesionales que no saben que van a hacer ni donde ubicarse, dado que no se ha contado ni se cuenta a la fecha con un Plan de Desarrollo de la educación superior universitaria, el cual implique el direccionamiento de la demanda de manera exógena ante una oferta endógena (quantum de vacantes y sus modalidades).

Contamos al 31/12/2006 con cerca de 28 millones de habitantes, con alrededor de 559,149 alumnos universitarios (sistema macro universitario), 35 Universidades públicas y 56 universidades privadas, las que en su mayoría han sido creadas siguiendo un ánimo de lucro y de interés políticos, muy distante de los reales requerimientos de un verdadero desarrollo de la educación superior.

Se tiene que para el año 2004 / 2006 un total de 471 facultades, con 1153 carreras profesionales, distribuidas en 663 para las universidades públicas y 490 para las universidades privadas, las carreras profesionales con más escuelas son las de contabilidad 61 , administración 61 , educación secundaria 53, derecho 53, enfermería y 55 de ingeniería de sistemas (e allí la respuesta de sobre los miles de profesionales de estas especialidades 
La demanda de educación universitaria estatal (DEUS) una apreciación para el periodo 1980-2006

desempleados y subempleados).

Universidades muchas de las operan con docentes (catedráticos), con un nivel de preparación y sin mayor experiencia en Docencia Universitaria, de allí también una falta de competitividad de sus egresados (no nos rasguemos las vestiduras con una realidad evidente).

Llama la atención que tanto el Ministerio de Educación, la Asamblea Nacional de Rectores, ni las propias Universidades hayan planteado una propuesta coherente referente al Desarrollo de la educación superior universitaria, no habiendo ninguna evaluación seria al respecto a fin de que esta problemática sea puesta en su real dimensión dada sus contradicciones y perjuicios.

Se han constituido Universidades y saturado con facultades del área de letras, por el facilismo de su constitución dejando de lado las de ciencias, física, química, biología, etc., por su implementación que es más costosa al demandar estos laboratorios complejos.

Con respecto a las Universidades Privadas, se controlan a través del Ministerio de Educación y la Asamblea Nacional de Rectores, las que les otorgan su aprobación oficial y su acreditación convirtiéndose en universidades de competencia con las nacionales adecuando, de manera más dinámica su infraestructura, personal académico, carreras, planes curriculares a la demanda que presenta el mercado de trabajo logrando una preferencia de sus egresados.

Hoy en día enfrentamos a un sistema económico global muy competitivo que demanda nuevas disciplinas, por lo que la falta de políticas en este sentido, de las organizaciones que dirigen y ordenan el sistema universitario nacional, mediante la propuesta de nuevas profesiones postergan nuestra participación en el sistema global de la economía.

\section{BIBLIOGRAFÍA}

SANTIAGO AUGURTO CALVO : "Problemática Universitaria". Imprenta Universidad Federico Villarreal - Lima, 1999.

ROSARIO YSLADO MÉNDEZ : "Sistema Universitario Internacional y Nacional" Editorial San Marcos - Diciembre, 2003

PEDRO FRANCK BALLVE : "La Educación Publica, Los Pobres y El Ajuste" - Romi Maqui Editores SRL - 1995 


\section{Ysac Ospino Edery}

ANIBAL SÁNCHEZ AGUILAR : "Visión y Dimensión de la Universidad Peruana, un Siglo de desarrollo universitario en el Perú 1990 - 2000". Editorial San Marcos-2001

HERNANDO DE SOTO : "El otro sendero". Instituto Libertad y Democracia, Sexta Edición, Marzo - 1987.

PEDRO PARIONA M. : "Universidad Peruana, Origen Desarrollo y Perspectivas". Ediciones Vientos Nuevos - Abril, 2001.

"EDUCACIÓN SUPERIOR EN EL PERÚ": Datos para el análisis Documentos de trabajo. GRADE, Septiembre, 1990

RESUMEN ESTADÍSTICO - 1980/1990/2000/2004. Dirección de Planificación Universitaria, Dirección de Estadística Informativa, Asamblea Nacional de Rectores.

POBREZA Y ECONOMÍA SOCIAL : Análisis de una encuesta (ENNIV - 1997) Instituto Cuanto-Agosto, 1999

NEMESIO ESPINOZA HERRERA : "Gerencia Universitaria". Editorial San Marcos Agosto, 2000

CARLOS SÁNCHEZ RAMÍREZ : "Ensayo de Epistemología Educativa". Editorial San Marcos-Diciembre 1999

Direcciones Web : Instituto Nacional de Estadística e Informática: www.inei.gob.pe, http://escale.minedu.gob.pe

MARIO BUNGE : Investigación Científica. Editorial Ariel - Barcelona, Edición 2000

\section{NOTAS AL PIE DE PAGINA}

1. Diario la Razón, 21 de Noviembre del 2006

2. "Causas de agravamiento de la pobreza en el Perú desde fines de la década de los 80's" - Francisco Verdera. PUC. 